\title{
Im Dienste der klinischen Neurowissenschaften
}

\author{
Interview: Isabel Zwyssig
}

M.A., koordinierende Redaktorin

Nicht nur möchte die Swiss Federation of Clinical Neuro-Societies (SFCNS) die öffentliche Wahrnehmung der Neurowissenschaften und deren Bedeutung für das Gesundheitswesen stärken, sondern auch die Zusammenarbeit zwischen einzelnen neurologischen Fachgesellschaften verbessern. Prof. Dr. med. Anton Valavanis und Prof. Dr. med. Claudio L.A. Bassetti stellen die Organisation vor.

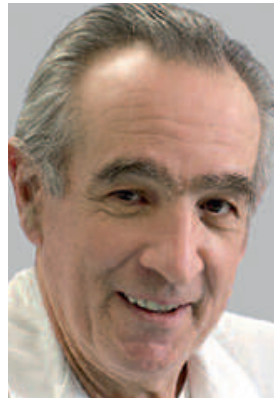

Prof. Dr. med. Anton Valavanis, aktueller Präsident der SFCNS seit 2013, Direktor des Instituts für Neuroradiologie, Leiter des Klinischen Neurozentrums, UniversitätsSpital Zürich

Die SFCNS sieht sich als Verein zur Förderung der klinischen Neurowissenschaften. Warum wurde der Verein gegründet?

Anton Valavanis: Die Idee zur Gründung einer Dachorganisation für die Fachgesellschaften, welche die klinischen Neurodisziplinen vertreten, ist anlässlich eines «Brainstorming»-Meetings, das am 30. August 2006 auf Initiative von Prof. Claudio L.A. Bassetti am Bahnhofbuffet Zürich stattfand, nach einer mehrstündigen Beratung entstanden. Am Treffen teilgenommen haben auch die damaligen Präsidenten der Schweizerischen Neurologischen Gesellschaft, Prof. Christian Hess, der Schweizerischen Gesellschaft für Neurochirurgie, Prof. Gerhard Hildebrandt und der Schweizerischen Gesellschaft für Neuroradiologie, Prof. Anton Valavanis. Die Teilnehmer waren der Überzeugung, dass der Superspezialisierung und der damit verbundenen wachsenden Fragmentierung der Neurofächer entgegengewirkt werden sollte, indem man Kommunikation und Kooperation zwischen Spezialisten stärkt. Dies einerseits um eine synthesefähige (holistische) Betrachtung der Neuromedizin auch in der $\mathrm{Zu}$ kunft zu sichern, anderseits auch um übergeordnete interdisziplinäre, gemeinsame Ziele besser erreichen zu können.

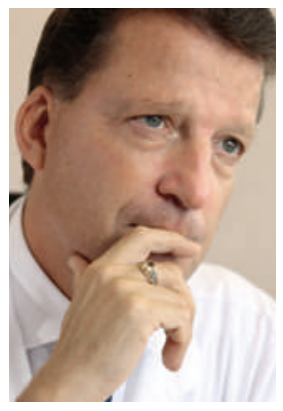

Prof. Dr. med. Claudio L.A. Bassetti, erster Präsident der SFCNS von 2009 bis 2013, Klinikdirektor und Chefarzt, Universitätsklinik für Neurologie, Inselspital Bern Leiter und Mitgründer des Neurozentrums, Inselspital Bern, Gründer und erster Leiter (2009-2012) des Neurocentro della Svizzera Italiana.

Wie reagierte die neurowissenschaftliche Gemeinschaft auf die Idee der Vereinsgründung?

Anton Valavanis: Die Idee wurde von der klinischneurowissenschaftlichen Gemeinschaft positiv aufgenommen. Mit administrativer und organisatorischer Unterstützung durch das Institut für Medizin und Kommunikation AG wurden in der Folge unter den Vertretern der Schweizerischen Gesellschaften für Neurologie, Klinische Neurophysiologie, Neurochirurgie, Neuroradiologie, Neuropädiatrie und Neuropathologie die Statuten erarbeitet. Am 27. Januar 2009 wurde der Verein gegründet. Mittlerweile haben sich der SFCNS 14 Gesellschaften aus den klinischen Neurowissenschaften angeschlossen.

\section{Können Sie die wichtigsten Ziele des Vereins nennen?} Claudio L.A. Bassetti: Die SFCNS verfolgt als Hauptziele, die klinischen Neurowissenschaften in der Schweiz zu fördern, die interdisziplinäre Zusammenarbeit mit neurowissenschaftlichen Organisationen, Standesorganisationen, politischen Instanzen und Kostenträgern zu stärken, die Weiter- und Fortbildung auf dem Gesamtgebiet der klinischen Neurowissenschaften zu unterstützen und Weiter- und Fortbildungsveranstaltungen auf hohem Niveau zu organisieren. 


\section{Welche Hauptanliegen vertritt die SFCNS gegen aussen?}

Anton Valavanis: Die Fortschritte, welche in den letzten Jahrzehnten auf den Gebieten der Hirnforschung und der klinisch-neurologischen Wissenschaften erzielt wurden, erklären das grosse, öffentliche Interesse, welches diese in der Gesellschaft und der Politik finden. Die Neurowissenschaft mit ihren grundlagenwissenschaftlichen und klinischen Komponenten ist eine neue Leitdisziplin an der Schnittstelle zwischen Medizin und Naturwissenschaft geworden. Es ist ein Hauptanliegen der SFCNS einerseits durch angemessene Öffentlichkeitsarbeit die Wahrnehmung der Neurowissenschaften und deren Bedeutung für das Gesundheitswesen zu stärken und andererseits die Zusammenarbeit mit der Swiss Society for Neuroscience, welche die grundlegenden neurowissenschaftlichen Disziplinen vertritt, an den klinisch und translational relevanten Schnittstellen auszubauen.

Werden diese Anliegen von den einzelnen Fachgesellschaften denn nicht mit genügend Gewicht vertreten? Anton Valavanis: Diese Anliegen werden natürlich von den einzelnen Fachgesellschaften auch vertreten, aber verständlicherweise primär aus der Perspektive der Fachrichtung jeder einzelnen Gesellschaft. Die SFCNS bündelt diese Anliegen, berücksichtigt dabei deren Interaktion und sucht die Unterstützung bei allen in ihr vereinten Gesellschaften, was schliesslich dem jeweiligen Anliegen oder Vorstoss mehr Gewicht bzw. Wirksamkeit verleiht.

\section{Welches waren die grössten Schwierigkeiten im Zusammenhang mit der Gründung und Etablierung der SFCNS?}

Claudio L.A. Bassetti: Erfreulicherweise waren sowohl die Gründung wie auch die anschliessende Etablierung, die noch im Gang ist, nicht von Schwierigkeiten oder Konflikten innerhalb der beteiligten Gesellschaften gekennzeichnet. Dies bestätigt, dass die damalige Entscheidung, die SFCNS zu gründen, sinnvoll war, weil sie einem gemeinsamen Bedürfnis entsprach.

\section{Wo liegen - abgesehen von der Repräsentation der klinischen Neurowissenschaften gegen aussen - weitere Schwerpunkte der Vereinstätigkeit?}

Claudio L.A. Bassetti: Ein wichtiger Schwerpunkt und zugleich eine Herausforderung der SFCNS ist die Organisation des alle drei Jahre stattfindenden Kongresses. Er bildet eine Plattform für den Wissensaustausch und ermöglicht die Anbahnung oder Vertiefung von interdisziplinären Kooperationen zwischen Kollegen oder Institutionen der klinischen Neurodisziplinen aus dem
In- und Ausland. Am SFCNS-Kongress nehmen jeweils etwa 1000 Ärztinnen und Ärzte teil. Der erste Kongress fand 2010 in Basel, der zweite 2013 in Montreux statt und der dritte wird 2016 in Basel abgehalten werden.

\section{Wie positioniert sich die SFCNS in Bezug auf hochspezialisierte Medizin?}

Anton Valavanis: Die Umsetzung der Beschlüsse der GDK im Rahmen der interkantonalen Vereinbarung zur hochspezialisierten Medizin (IV-HSM), welche die klinischen Neurowissenschaften betreffen, ist ein weiterer, sehr anspruchsvoller Schwerpunkt der SFCNS. Es sind dies die Entscheide zur Planung der hochspezialisierten Medizin (HSM) im Bereich der hochspezialisierten Behandlung von Hirnschlägen (im Rahmen von sog. Stroke Centers und Stroke Units), der chirurgischen Behandlung der refraktären Epilepsie beim Erwachsenen, der stereotaktischen Chirurgie der anormalen, ungewollten Bewegungen und tiefen Hirnstimulation (Deep Brain Stimulation) beim Erwachsenen, der neurochirurgischen Behandlung von vaskulären Erkrankungen des zentralen Nervensystems (ZNS) ohne die komplexen vaskulären Anomalien, der neurochirurgischen Behandlung von komplexen vaskulären Anomalien des zentralen Nervensystems (ZNS) und die Behandlung der seltenen Rückenmarkstumoren.

Ein wichtiger Erfolg für die SFCNS war die Übertragung der Mandate durch die GDK zur Zertifizierung bzw. Anerkennung der zugeteilten Zentren. Damit wurde die SFCNS auch von gesundheitspolitischer Seite als Repräsentantin der klinischen Neurodisziplinen anerkannt.

\section{Was haben Sie in diesen Bereichen bisher erreicht?} Anton Valavanis: Die bisherigen SFCNS-Kongresse waren dank der Auswahl interdisziplinärer Themen, der Beteiligung herausragender Referenten, einer ausgewogenen Balance zwischen neurowissenschaftlicher Ausrichtung und Praxisorientierung, der Zurverfügungstellung von Plattformen für die aktive Teilnahme des Nachwuchses und mit einer Teilnahme von jeweils über 1000 Ärzten und Neurowissenschaftlern sehr erfolgreich. Damit hat sich der SFCNS-Kongress als eine zentrale Institution der klinischen Neurowissenschaften etabliert.

Claudio L.A. Bassetti: Der aufwendige Prozess der Zertifizierung bzw. Anerkennung der im Rahmen der GDK-Entscheide zur IV-HSM zugeteilten Zentren sind für alle sechs Mandate angelaufen. Bereits wurden alle neun Schlaganfallzentren der Schweiz und neun Stroke Units durch die SFCNS erfolgreich zertifiziert. Die übrigen Mandate befinden sich in unterschiedlichen Umsetzungsphasen. Bereits jetzt kann man sagen, dass die SFCNS eine geeignete Plattform zur 
Umsetzung dieser fachlich und politisch ehrgeizigen Vorhaben im Bereich der klinischen Neurodisziplinen ist. Im Rahmen zahlreicher Sitzungen und bilateraler Gespräche konnte disziplinenübergreifend ein breites Bewusstsein und Akzeptanz für die Zentrumsbildung und Qualitätssicherung geschaffen werden. Dennoch bleiben bei Fachkollegen Fragen offen; eine kontinuierliche auch öffentliche Diskussion wird weiterhin wichtig und notwendig sein.

\section{Was bedeuten diese Erfolge für Ihre zukünftige Arbeit?}

Anton Valavanis: Diese ersten Erfolge stärken die Position der SFCNS im medizinischen, standes- und gesundheitspolitischen Umfeld und bilden eine solide Basis für die weitere Arbeit zur Erreichung der angestrebten Ziele.

\section{Wo sehen Sie die wichtigsten Herausforderungen für Ihre Organisation?}

Claudio L.A. Bassetti: Wir erkennen mehrere Bereiche, in welchen die SFCNS als Organisation für die Zukunft der Neurofächer eine wichtige Funktion wahrnehmen könnte:

1) Die Positionierung der Neurofächer zwischen klinischer Versorgung und Neurowissenschaft. Die SFCNS fördert u.a. mit ihren grossen Kongressen die Interaktion in Lehre und Forschung unter den Neuroklinikern, aber speziell auch zwischen den klinischen und Grundlagefächern.

2) Die Betreuung von Patienten mit Erkrankungen des Nervensystems, von der Notfallaufnahme bis zur Spitalentlassung bzw. Neurorehabilitation, verlangt eine multidisziplinäre Zusammenarbeit zwischen den verschiedenen Neurospezialisten. Allerdings werden dadurch die traditionellen Grenzen der Fächer "gesprengt». Deshalb braucht es eine (neue) Kultur und eine (gemeinsame) Sprache der Zusammenarbeit, wofür sich die SFCNS seit Jahren stark einsetzt. Die Schaffung von sog. Neurozentren in der Schweiz (in Lausanne und im Tessin, neuerdings auch in Bern und Zürich) wurde mit der gleichen Zielsetzung initiiert.

3) Die weitere Entwicklung der Neurofächer, von der Grundversorgung bis zu den Bereichen der hochspezialisierten Medizin, vom Kindes- bis zum Greisenalter, inkl. der Neurorehabilitation und der Palliativmedizin, wird ebenfalls von der Präsenz der SFCNS und durch ihre Unterstützung profitieren - sowohl auf fachlicher als auch auf politischer Ebene.

\section{Inwiefern profitiert die Schweizer Ärzteschaft vom Engagement der SFCNS?}

Anton Valavanis: Die Schweiz hat eine lange Tradition auf dem Gebiet der klinischen Neurowissenschaften. Schweizer Ärzte haben wegweisende und herausragende Beiträge zur Entwicklung der Neurowissenschaften geleistet. Die Entwicklung der klinischen Neurowissenschaften hat zur Schaffung diverser Fachrichtungen, die sich in Fachgesellschaften organisiert haben, geführt. Allen gemeinsam ist die Beschäftigung mit einem besonderen Aspekt der Diagnostik und Behandlung von Erkrankungen oder Dysfunktionen des Zentralnervensystems. Sie wirken auf der Grundlage des hippokratischen neurozentrischen Konzeptes der Medizin.

Claudio L.A. Bassetti: Als Dachorganisation der klinisch-neurowissenschaftlichen Gesellschaften mit Engagement in der Vertretung der übergeordneten standes- und gesundheitspolitischen Interessen ihrer Mitgliedgesellschaften, in der Unterstützung der Anliegen ihrer Mitgliedgesellschaften in den Belangen der Weiter- und Fortbildung sowie mit ihrer Öffentlichkeitsarbeit stellt sie einen zuverlässigen Ansprechpartner für die Schweizer Ärzteschaft dar. Darüber hinaus trägt sie zur Steigerung der Attraktivität der klinischen Neurodisziplinen für den ärztlichen Nachwuchs in der Schweiz bei.

\section{Welche langfristigen Ziele möchten Sie realisieren?}

Claudio L.A. Bassetti: Wichtige langfristige Ziele der SFCNS sind 1) die Attraktivität der Neurofächer für Mediziner und Neurowissenschaftler und speziell für den Nachwuchs durch eine konsequente Förderung der Inter- und Multidisziplinarität in der Lehre sowie durch ein klares "Commitment» zur klinischen und translationalen Forschung nachhaltig zu erhöhen, 2) die gemeinsame Standespolitik für die klinischen Neurowissenschaften weiterzuentwickeln, um damit ihre Positionierung zu stärken, 3) die Qualitätssicherung u.a. durch Zertifizierungen der Neurozentren auszubauen. In diesem Sinne könnte die SFCNS auch ein Vorbild darstellen für andere multidisziplinär zusammengesetzte medizinische Bereiche.

\section{Bildnachweis}

Porträt Bassetti: photo by rémy steinegger / copyright by www.steineggerpix.com 